\title{
MULTIPLE SCATTERING OF SH WAVES BY AN EMBEDDED TRUNCATED CIRCULAR CAVITY
}

\author{
Deng-How Tsaur \\ Department of Harbor and River Engineering, National Taiwan Ocean University, Keelung, Taiwan., \\ hretdh@ntou.edu.tw \\ Kao-Hao Chang \\ Department of Harbor and River Engineering, National Taiwan Ocean University, Keelung, Taiwan.
}

Follow this and additional works at: https://jmstt.ntou.edu.tw/journal

Part of the Civil and Environmental Engineering Commons

\section{Recommended Citation}

Tsaur, Deng-How and Chang, Kao-Hao (2012) "MULTIPLE SCATTERING OF SH WAVES BY AN EMBEDDED TRUNCATED CIRCULAR CAVITY," Journal of Marine Science and Technology. Vol. 20: Iss. 1, Article 9. DOI: $10.51400 / 2709-6998.2424$

Available at: https://jmstt.ntou.edu.tw/journal/vol20/iss1/9

This Research Article is brought to you for free and open access by Journal of Marine Science and Technology. It has been accepted for inclusion in Journal of Marine Science and Technology by an authorized editor of Journal of Marine Science and Technology. 


\title{
MULTIPLE SCATTERING OF SH WAVES BY AN EMBEDDED TRUNCATED CIRCULAR CAVITY
}

\author{
Deng-How Tsaur and Kao-Hao Chang
}

Key words: scattering, SH waves, truncated circular cavity.

\begin{abstract}
The region-matching technique is implemented to derive a series solution to the $\mathrm{SH}$-wave scattering problem of an embedded truncated circular cavity. An introduced auxiliary boundary partitions the whole analyzed region into two subregions, whose displacement fields are expressed as a superposition of appropriate wavefunctions. Taking advantage of the method of images, the zero-stress boundary condition on the horizontal ground surface is satisfied automatically. Suitable Graf's addition formulas facilitate the enforcement of boundary conditions on the artificial interface and curved cavity surface for determining the expansion coefficients. Effects of several parameters on steady-state surface motions are discussed. Transient changes in underground motion are also included. Comparisons with published data for circular cases show good agreement. The present study not only gives a theoretical groundwork for practical unlined tunnels, but also can be applicable to the earthquake-resistant design methods of underground structures.
\end{abstract}

\section{INTRODUCTION}

Scattering and diffraction of natural and artificial subsurface cavities have long been a topic of considerable interest to geophysicists, seismologists and engineers. This is because they are highly related to some applications such as mineral prospecting, geological mapping, site characterization, concealed opening detection, defect location, and military use. In a broad sense, the embedded cavities have a main feature, that is, the complete enclosure in the host medium. This trait, from the seismological point of view, makes their dynamic behavior distinct from those of local free-surface topographies (e.g. canyons and hills) and near-surface geologic formations (e.g. sediment-filled valleys).

Paper submitted 02/05/10; revised 06/11/10; accepted 09/28/10. Author for correspondence: Deng-How Tsaur (e-mail: hretdh@ntou.edu.tw).

Department of Harbor and River Engineering, National Taiwan Ocean University, Keelung, Taiwan.
Since the 1980s, there have been many studies [2-4, 7-11, $13,15,17,23$ ] concerning the possible modification of surface ground motions elicited by dynamic sources in a half-plane containing a single cavity or multiple cavities. Most of these investigations focus on the cavities of circular or elliptic shapes. However, less attention was given to those of 'truncated' circular shape. For the purpose of fundamental studying, a practical tunnel, like the Qinling tunnel in Shaanxi Province of China (see Fig. 4 of [12]) and the shield tunnel in Tokyo Bay of Japan (see Fig. 6 of [20]), may be simply idealized as an embedded unlined tunnel of truncated circular cross-section. Although such a shape looks quite simple, the corresponding boundary-value problem cannot be solved directly by using the method of separation of variables because of the unconformity between the cavity surface and any one of separable coordinate systems. It is well known that only a few simple geometric shapes allow one to get complete expressions in terms of corresponding wavefunctions [21]. For the 2-D full-plane cavity problems pertaining to the simplest SH-wave incidence, Pao and Mow [14] have given a sequence of analytical solutions in their monograph. As to the half-plane cavity problems, even for the purest geometries (such as a circle and an ellipse), their elegant analytical solutions (e.g. explicit analytical determination of all basis coefficients in the wavefunction expansions) seem to be unavailable from published references. Fortunately, two series solutions under plane-wave incidence have been given by Lee [8] and Lee et al. [9] for the case of a circular cavity and for that of a semi-circular canyon with a subsurface circular cavity, respectively. Recently, Lee and Kallivokas [7] seemed to not take notice of the work done by Lee [8], and therefore followed similar lines to derive a series solution for an immersed circular cavity. Very recently, Smerzini et al. [17] presented a series solution for an embedded circular cavity upon planewave and line-source excitations. Indeed, these series solutions redeem the regret for the lack of elegant analytical solutions.

In the present work, carrying out the derivation of a series solution to the SH-wave scattering problem of an embedded truncated circular cavity is the authors' aim. Thus, the canonical circular geometries studied in the literature are broadened herein to cover a range of truncated circular shapes. Based on 
the success of deriving series solutions to the scattering problems of a truncated semi-circular canyon [18] and a partially filled semi-circular alluvial valley [19], the promising region-matching technique is adopted and extended herein again. As a basic problem in elastodynamics, the proposed simplified model is of great significance due to its inherent shape very close to that of a practical underground unlined tunnel. The following formulization constitutes a rigorous theoretical analysis, and produces useful results, giving us an insight into the dynamic response and wave propagation behaviour around an embedded truncated circular cavity. Besides, the present work is the groundwork for studying the case of an embedded polygonal cavity.

\section{THEORETICAL FORMULATION}

Consider a truncated circular cavity buried in a homogeneous, isotropic, linearly elastic half-plane (with shear modulus $\mu$ and shear wave velocity $c_{s}$ ). An infinite train of unit-amplitude plane SH waves (with angular frequency $\omega$ ) is incident upon this system at an angle $\alpha$ to the $y$-axis. The geometric layout of the problem is illustrated in Fig. 1. The cross-section of the cavity is basically circular in shape but horizontally truncated at its lower part. The auxiliary boundary $S_{I}$ (which is part of a circular arc with central angle $2 \beta$ ) and the curved cavity surface form a circle with radius $a$. The cavity depth $d$ is defined as the distance from the ground surface to the top rim of cavity. The truncation thickness $h$ is defined as the distance from the unreal circular-arc cavity bottom to the real flat one. The center of a circular image cavity is located at a distance $d+a$ above the ground surface. Four Cartesian and four cylindrical coordinate systems are employed. In the following, the authors mainly focus on the cases of circles, major segments and semicircles (i.e. $0 \leq h / a \leq$ 1 ), which can be referred to most practical cases of shield tunnels.

As seen in Fig. 1, by introducing a circular-arc auxiliary boundary $S_{I}$, the half-plane is divided into two regions, an open region (1) and an enclosed region (2). The steady-state out-of-plane motions $u_{j}$ (the subscripts, 1 and 2, designate the total displacement fields in regions (1) and (2), respectively) are required to satisfy the governing Helmholtz equations. The harmonic time dependence is given by a factor $\exp (i \omega t)$ and suppressed throughout this paper.

In region (1), the zero-stress boundary conditions on the left- and right-hand horizontal ground surfaces are

$$
\tau_{\theta z}^{(1)}=\frac{\mu}{r} \frac{\partial u_{1}}{\partial \theta}(r, \theta)=0, \text { for } \theta= \pm \frac{\pi}{2}
$$

and the traction-free boundary condition on the curved cavity surface is

$$
\tau_{r_{1} z}^{(1)}=\mu \frac{\partial u_{1}}{\partial r_{1}}\left(r_{1}, \theta_{1}\right)=0, \text { for } r_{1}=a \text { and } \beta \leq \theta_{1} \leq 2 \pi-\beta
$$

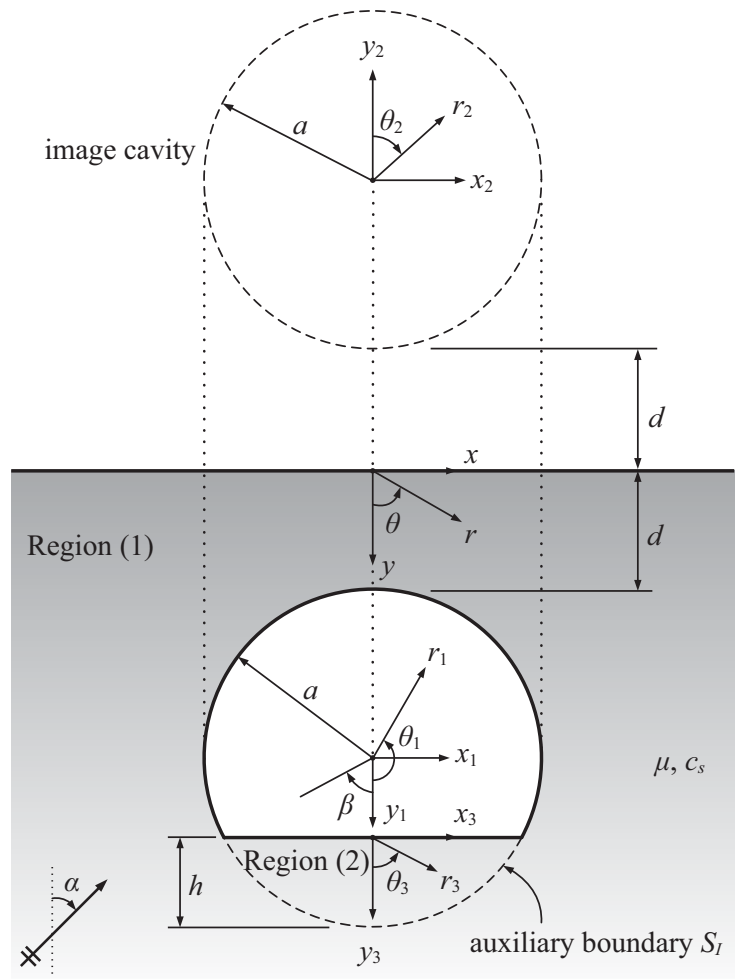

Fig. 1. Definition sketch for an embedded truncated circular cavity.

In region (2), the stress-free boundary conditions on the flat cavity surface are

$$
\tau_{\theta_{3} z}^{(2)}=\frac{\mu}{r_{3}} \frac{\partial u_{2}}{\partial \theta_{3}}\left(r_{3}, \theta_{3}\right)=0, \text { for } r_{3}<a \sin \beta \text { and } \theta_{3}= \pm \frac{\pi}{2}
$$

On the auxiliary boundary $S_{I}$, two matching conditions, which assure the continuity of normal components of the displacement and stress fields between regions (1) and (2), are given by

$$
\begin{gathered}
u_{1}\left(r_{1}, \theta_{1}\right)=u_{2}\left(r_{1}, \theta_{1}\right), \text { for } r_{1}=a \text { and }\left|\theta_{1}\right| \leq \beta \\
\tau_{r_{1} z}^{(1)}\left(r_{1}, \theta_{1}\right)=\tau_{r_{1} z}^{(2)}\left(r_{1}, \theta_{1}\right), \text { for } r_{1}=a \text { and }\left|\theta_{1}\right| \leq \beta
\end{gathered}
$$

For the problem under consideration, the application of the method of images is effective. By treating the ground surface as an ideal mirror, an image of the incident plane wave and an image cavity are both introduced. This profits the later construction of appropriate wavefunctions for satisfying the boundary conditions on the ground surface. At present, the original problem to be solved is converted into an equivalent two-scatterer problem.

In open region (1), adding the incident and reflected fields together (the latter is a mirror image of the former), the free wavefield $u^{F}$ in terms of the local coordinate system $\left(r_{1}, \theta_{1}\right)$ is of the form: 


$$
u^{F}\left(r_{1}, \theta_{1}\right)=\exp \left[i k r_{1} \cos \left(\theta_{1}+\alpha\right)\right]+\xi \cdot \exp \left[-i k r_{1} \cos \left(\theta_{1}-\alpha\right)\right]
$$

where $k=\omega / c_{s}$ is the shear wavenumber and $\xi=\exp (-2 i k d \cos$ $\alpha$ ) is the phase factor. Then, Eq. (6) can be expanded as [1, 22]:

$$
\begin{aligned}
u^{F}\left(r_{1}, \theta_{1}\right)= & \sum_{n=0}^{\infty} \varepsilon_{n}\left[i^{n}+\xi(-i)^{n}\right] J_{n}\left(k r_{1}\right) \cos n \alpha \cos n \theta_{1} \\
& +\sum_{n=1}^{\infty} \varepsilon_{n}\left[-i^{n}+\xi(-i)^{n}\right] J_{n}\left(k r_{1}\right) \sin n \alpha \sin n \theta_{1}
\end{aligned}
$$

where $\varepsilon_{n}$ is the Neumann factor (which is equal to 1 if $n=0$ and to 2 if $n \geq 1)$ and $J_{n}(\cdot)$ denotes the $n$th order Bessel function of the first kind.

When the incident wave and its mirror reflection impinge, respectively, upon the cavity and its image, they give rise to scattered waves. The resultant scattered field $u^{S}$ is the sum of the two scattering components propagating radially outward from the centers of the cavity and its image, respectively. Its proper wavefunction, satisfying the Helmholtz equation and Sommerfeld radiation condition at infinity, can be written as

$$
\begin{aligned}
u^{S}= & \sum_{n=0}^{\infty} A_{n}\left[H_{n}^{(2)}\left(k r_{1}\right) \cos n \theta_{1}+H_{n}^{(2)}\left(k r_{2}\right) \cos n \theta_{2}\right] \\
& +\sum_{n=1}^{\infty} B_{n}\left[H_{n}^{(2)}\left(k r_{1}\right) \sin n \theta_{1}+H_{n}^{(2)}\left(k r_{2}\right) \sin n \theta_{2}\right]
\end{aligned}
$$

where $H_{n}^{(2)}(\cdot)$ are the $n$th order Hankel functions of the second kind, and the complex expansion coefficients $A_{n}$ and $B_{n}$ are unknown. From Eqs. (7) and (8), one can find that they automatically satisfy the zero-stress condition on the horizontal ground surface, Eq. (1), as a natural consequence of the symmetry of the equivalent two-object scattering system.

The union of the free wavefield $u^{F}$ and the resultant scattered wavefield $u^{S}$ gives the total wavefield $u_{1}$ in region (1), that is,

$$
u_{1}=u^{F}+u^{S}
$$

In enclosed region (2), the wavefield $u_{2}$, satisfying the Helmholtz equation and the boundary conditions on the flat cavity surface, Eq. (3), can be expressed as

$$
\begin{aligned}
u_{2}\left(r_{3}, \theta_{3}\right)= & \sum_{n=0}^{\infty} C_{n} J_{2 n}\left(k r_{3}\right) \cos (2 n) \theta_{3} \\
& +\sum_{n=0}^{\infty} D_{n} J_{2 n+1}\left(k r_{3}\right) \sin (2 n+1) \theta_{3}
\end{aligned}
$$

where the coefficients $C_{n}$ and $D_{n}$ are to be determined.

Next, in order to match the boundary conditions across the auxiliary interface $S_{I}$, the required coordinate translations for Bessel and Hankel functions are carried out via the Graf's addition formulas [22] (rewritten in suitable forms for our purpose). The defined function $U_{m, n}^{ \pm}(\cdot)$ used in the following Eqs. (12)-(15) is given by

$$
U_{m, n}^{ \pm}(\cdot)=\frac{\varepsilon_{m}}{2}\left[(-1)^{n} H_{m+n}^{(2)}(\cdot) \pm H_{m-n}^{(2)}(\cdot)\right]
$$

and another one $J_{m, n}^{ \pm}(\cdot)$ can be found in Eq. (16) of [18].

After re-expressing the resultant scattered wavefield $u^{S}$ and the wavefield $u_{2}$, applying the orthogonal properties of cosine functions to the stress continuity condition, Eq. (5), and the traction-free condition, Eq. (2), integrating over the range $[-\pi, \pi]$, rearranging, and truncating the infinite series to a finite number of terms, the following system of linear algebraic equations is obtained.

$$
\begin{gathered}
\sum_{n=0}^{N-1} A_{n}\left\{(-1)^{q} J_{q}^{\prime}(k a) U_{q, n}^{+}[2 k(d+a)]+\delta_{n, q} H_{q}^{(2)^{\prime}}(k a)\right\} \\
-\frac{\varepsilon_{q}}{2 \pi} \sum_{n=0}^{N-1} C_{n} \sum_{m=0}^{M} J_{m}^{\prime}(k a) J_{m, 2 n}^{+}[k(a-h)] I_{m, q}^{C}(\beta) \\
=-\varepsilon_{q}\left[i^{q}+\xi(-i)^{q}\right] \cos (q \alpha) J_{q}^{\prime}(k a), \\
q=0,1,2, \cdots, N-1
\end{gathered}
$$

where $I_{m, n}^{C}(\beta)$ can be found in Eq. (20) of [18].

Then, multiplying the displacement continuity condition, Eq. (4), by cosine functions, integrating over the range $[-\beta, \beta]$, rearranging and truncating the infinite series lead to the following system of equations,

$$
\begin{aligned}
\sum_{n=0}^{N-1} A_{n}\{ & H_{n}^{(2)}(k a) I_{n, q}^{C}(\beta) \\
& \left.+\sum_{m=0}^{M}(-1)^{m} J_{m}(k a) U_{m, n}^{+}[2 k(d+a)] I_{m, q}^{C}(\beta)\right\} \\
& -\sum_{n=0}^{N-1} C_{n} \sum_{m=0}^{M} J_{m}(k a) J_{m, 2 n}^{+}[k(a-h)] I_{m, q}^{C}(\beta) \\
=-\sum_{n=0}^{N-1} \varepsilon_{n}\left[i^{n}+\xi(-i)^{n}\right] \cos (n \alpha) J_{n}(k a) I_{n, q}^{C}(\beta), & q=0,1,2, \cdots, N-1
\end{aligned}
$$

Notice that, in Eqs. (12) and (13), the summation indexes $n$ are truncated to $N-1$ terms, $m$ are truncated to $M$ terms, and the indexes $q$ are selected to be $N-1$. Apparently, Eqs. (12) and (13) constitute a system of $2 N$ equations for unknown coefficients $A_{n}$ and $C_{n}$, and they can be solved simultaneously by standard matrix techniques.

In a similar manner, multiplying Eqs. (5), (2) and (4) by sine functions, integrating, rearranging, and truncating the 
infinite series result in the following system of $2 N$ equations for the unknowns $B_{n}$ and $D_{n}$,

$$
\begin{gathered}
\sum_{n=1}^{N} B_{n}\left\{(-1)^{q} J_{q}^{\prime}(k a) U_{q, n}^{-}[2 k(d+a)]+\delta_{n, q} H_{q}^{(2)^{\prime}}(k a)\right\} \\
+\frac{1}{\pi} \sum_{n=0}^{N-1} D_{n} \sum_{m=1}^{M} J_{m}^{\prime}(k a) J_{m, 2 n+1}^{-}[k(a-h)] I_{m, q}^{S}(\beta) \\
=-\varepsilon_{q}\left[-i^{q}+\xi(-i)^{q}\right] \sin (q \alpha) J_{q}^{\prime}(k a), \\
q=1,2,3, \cdots, N
\end{gathered}
$$

$$
\begin{aligned}
& \sum_{n=1}^{N} B_{n}\{ H_{n}^{(2)}(k a) I_{n, q}^{S}(\beta) \\
&+\left.\sum_{m=1}^{M}(-1)^{m} J_{m}(k a) U_{m, n}^{-}[2 k(d+a)] I_{m, q}^{S}(\beta)\right\} \\
&+\sum_{n=0}^{N-1} D_{n} \sum_{m=1}^{M} J_{m}(k a) J_{m, 2 n+1}^{-}[k(a-h)] I_{m, q}^{S}(\beta) \\
&=-\sum_{n=1}^{N} \varepsilon_{n}\left[-i^{n}+\xi(-i)^{n}\right] \sin (n \alpha) J_{n}(k a) I_{n, q}^{S}(\beta), \\
& q=1,2,3, \cdots, N
\end{aligned}
$$

where $I_{m, n}^{S}(\beta)$ can be found in Eq. (21) of [18].

Once the expansion coefficients $A_{n}, B_{n}, C_{n}$ and $D_{n}$ are obtained, all physical quantities of interest can be evaluated in a straightforward way.

When the truncation thickness $h$ is equal to zero (i.e. $\beta=0$ ), the truncated case turns into the circular one, and the unknown coefficients $\left(C_{n}\right.$ and $\left.D_{n}\right)$ of region (2) vanish directly. Also, Eqs. (12) and (14) reduce, respectively, to the following systems of equations,

$$
\begin{gathered}
\sum_{n=0}^{N-1} A_{n}\left\{(-1)^{q} J_{q}^{\prime}(k a) U_{q, n}^{+}[2 k(d+a)]+\delta_{n, q} H_{q}^{(2)^{\prime}}(k a)\right\} \\
=-\mathcal{E}_{q}\left[i^{q}+\xi(-i)^{q}\right] \cos (q \alpha) J_{q}^{\prime}(k a), \\
q=0,1,2, \cdots, N-1 \\
\sum_{n=1}^{N} B_{n}\left\{(-1)^{q} J_{q}^{\prime}(k a) U_{q, n}^{-}[2 k(d+a)]+\delta_{n, q} H_{q}^{(2)^{\prime}}(k a)\right\} \\
=-\varepsilon_{q}\left[-i^{q}+\xi(-i)^{q}\right] \sin (q \alpha) J_{q}^{\prime}(k a), \\
q=1,2,3, \cdots, N
\end{gathered}
$$

Once the scattering coefficients $A_{n}$ and $B_{n}$ are obtained, the total scattered wavefield can be completely determined. For this limiting case, results obtained by Eqs. (16) and (17) coincide with those of existing works [10, 13, 23]. Detailed comparisons will be shown in Section III.1.

It is convenient to define the dimensionless frequency $\eta$ as the ratio of the maximum width of the cavity to the incident wavelength $\lambda$, that is,

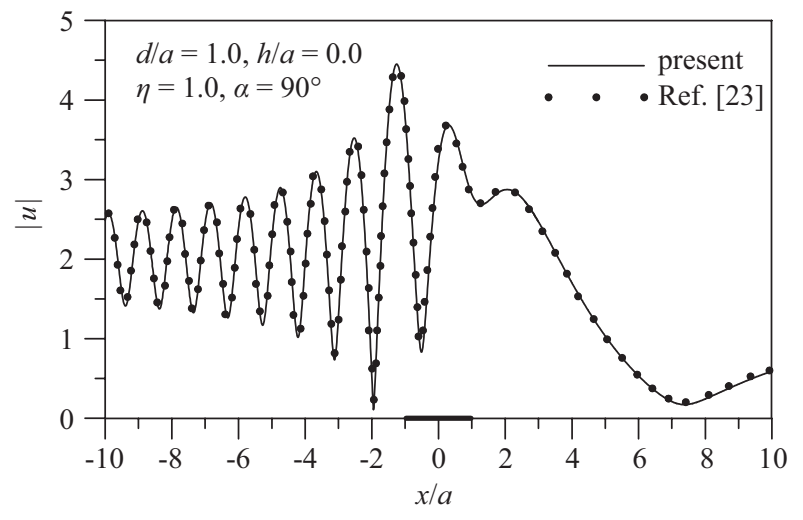

Fig. 2. Displacement amplitudes on the ground surface for comparison with the results of Ref. [23].

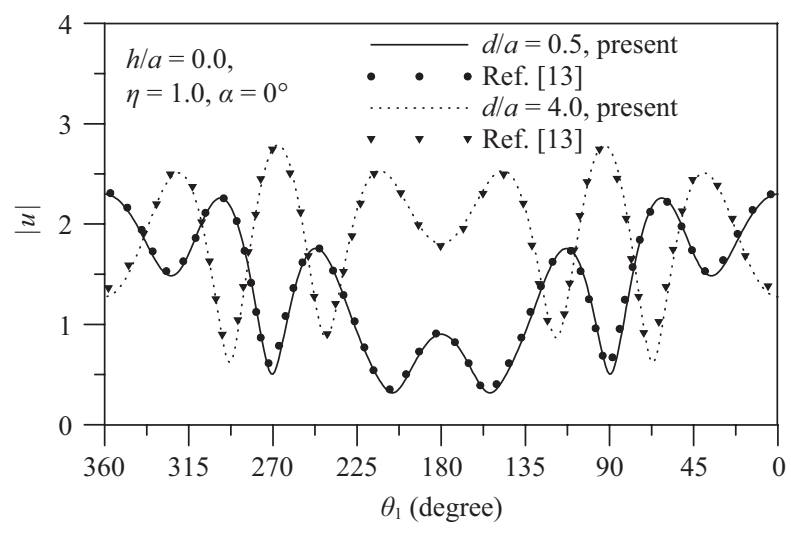

Fig. 3. Displacement amplitudes on the cavity surface for comparison with the results of Ref. [13].

$$
\eta=\frac{\omega a}{\pi c_{s}}=\frac{2 a}{\lambda}
$$

\section{NUMERICAL RESULTS AND DISCUSSIONS}

\section{Frequency-Domain Responses}

Convergence tests are carried out first to determine the truncation limit of the infinite series in Eqs. (12)-(15). A sequence of numerical experiments shows that the dimensionless frequency $\eta$ is the dominant factor affecting the convergence of surface displacement amplitudes (not the dimensionless cavity depth $d / a$ ). In general, more terms are required as the dimensionless frequency $\eta$ increases. In authors' experience, $M=200$ are adequate for $\eta \leq 16$.

\section{1) Validation for an Embedded Circular Cavity}

Concerning the case of an embedded circular cavity, former investigators have given various results helpful to checking the correctness of Eqs. (16) and (17). Initially, the case shown in Fig. 7 of [23] is computed in Fig. 2. Subsequently, two cases given in Fig. 2(d) of [13] are calculated in Fig. 3. From 


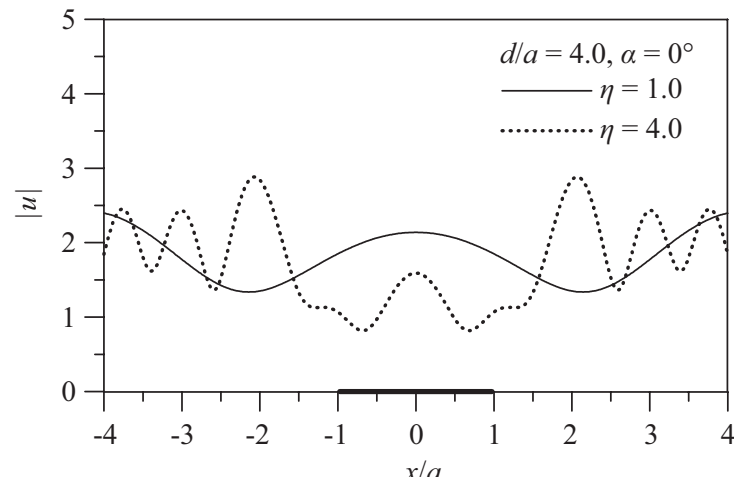

(a)

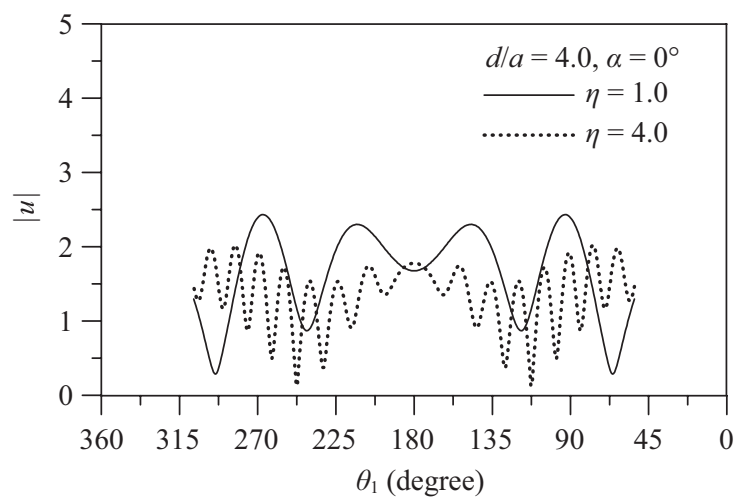

(b)

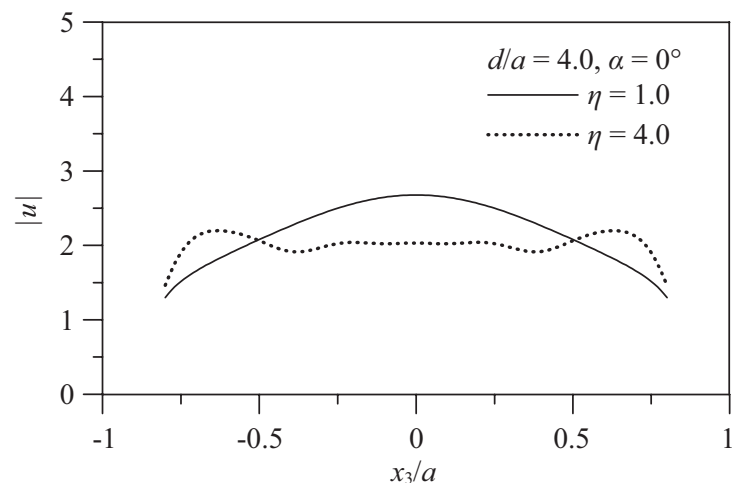

(c)

Fig. 4. Surface displacement amplitudes for $\eta=1.0$ and 4.0 with $d / a=4.0$ and $\alpha=0^{\circ}$. (a) displacement amplitudes on the ground surface, (b) displacement amplitudes on the curved cavity surface, and (c) displacement amplitudes on the flat cavity surface.

Figs. 2 and 3, good agreement can be seen between the present results and those shown in [23] and [13]. Besides, for other cases, such as those shown in Fig. 8 of [23], Fig. 4 of [10], and in Figs. 2 and 3 of [13], they are all in accordance with our results (not given here for reasons of brevity). Evidently, Figs. 2 and 3 not only support the validity of the present results and of those presented by earlier researchers, but also convince the authors that no mistakes are made in deriving Eqs. (16) and (17).

\section{2) Displacement Amplitudes on the Ground and Cavity Surfaces}

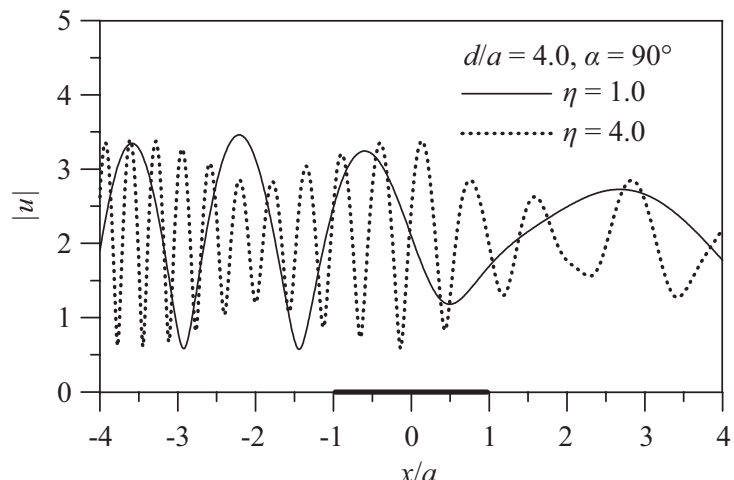

(a)

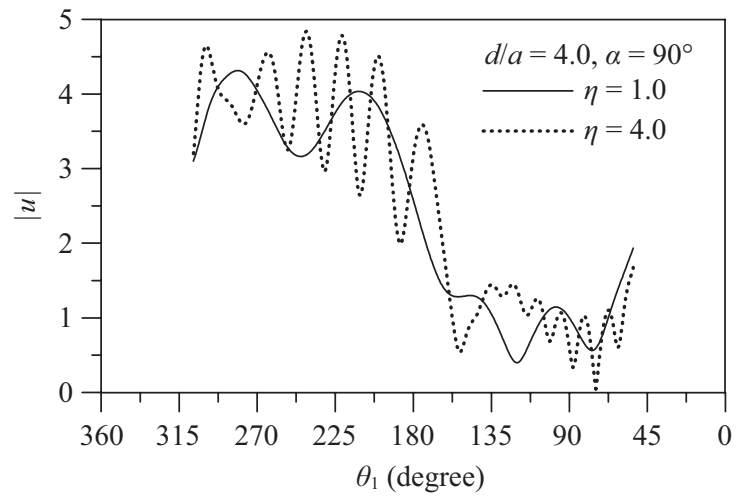

(b)

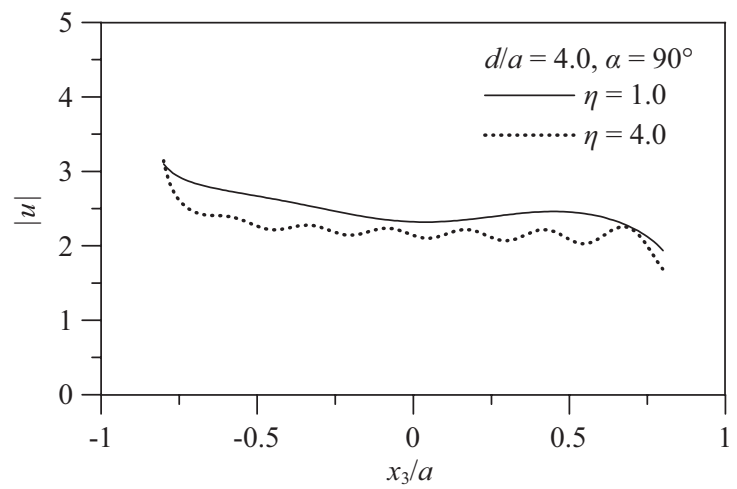

(c)

Fig. 5. Surface displacement amplitudes for $\eta=1.0$ and 4.0 with $d / a=4.0$ and $\alpha=90^{\circ}$. (a) displacement amplitudes on the ground surface, (b) displacement amplitudes on the curved cavity surface, and (c) displacement amplitudes on the flat cavity surface.

In order to exhibit the effect of the dimensionless frequency $\eta$ on the surface displacement amplitude $|u|$, the surface response calculations for $\eta=1.0$ and 4.0 are done. The dimensionless cavity depth $d / a$ is selected as 4.0 here, and this value is referred to the usual excavation depth for large railway tunnels in Japan (see, e.g. Fig. 1 of [6]). The dimensionless truncation thickness $h / a$ is chosen as 0.4. Corresponding results are shown in Figs. 4 and 5. For the curved cavity surface, the plotted range in the circumferential direction goes from $\theta_{1}=53.1^{\circ}$ to $306.9^{\circ}$.

For vertical incidence $\left(\alpha=0^{\circ}\right)$ in Fig. 4 , due to the sym- 


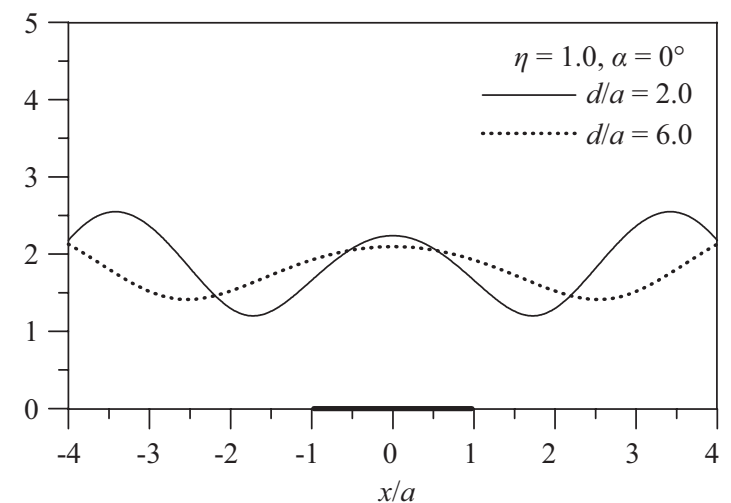

(a) $\eta=1.0$ and $\alpha=0^{\circ}$

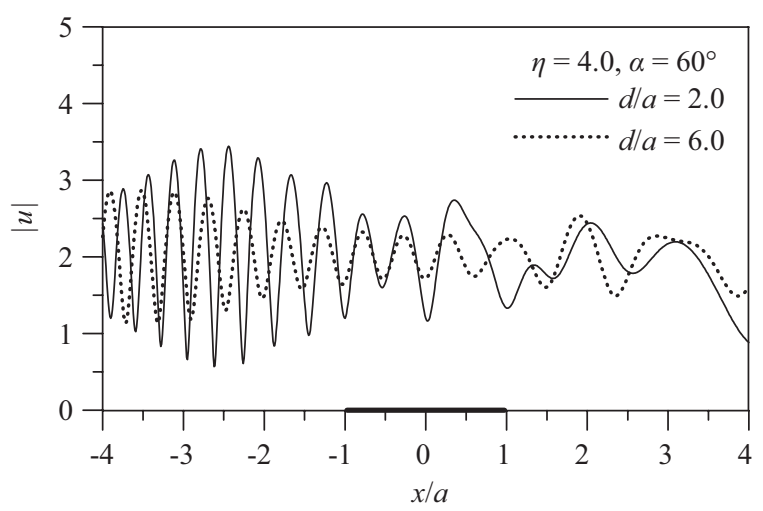

(c) $\eta=4.0$ and $\alpha=60^{\circ}$

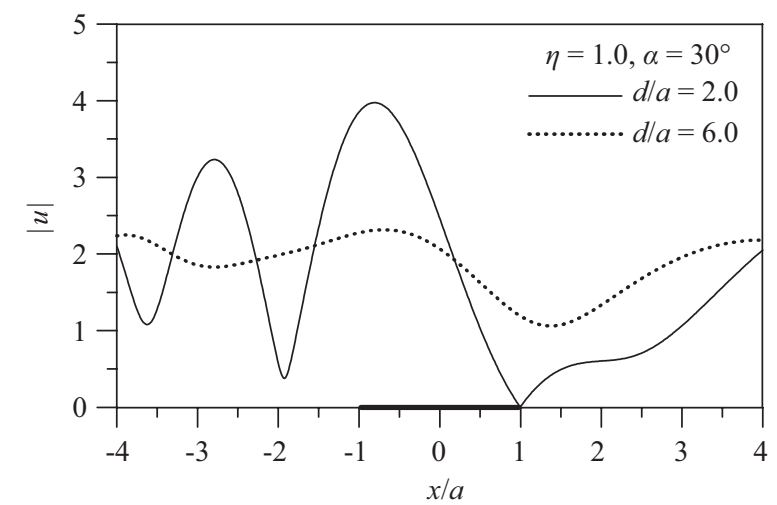

(b) $\eta=1.0$ and $\alpha=30^{\circ}$

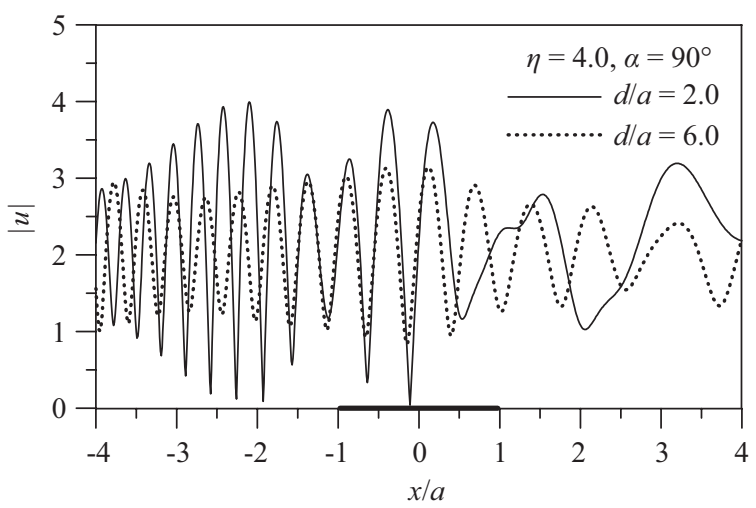

(d) $\eta=4.0$ and $\alpha=90^{\circ}$

Fig. 6. Displacement amplitudes on the ground surface for different dimensionless cavity depth $d / a$.

metry of the cavity, the symmetric feature of motions is expected. From Fig. 4(a), one can observe that, for the relatively low-frequency case $(\eta=1.0)$, the displacement amplitude on the ground surface over the cavity (i.e. $-1.0<x / a<1.0$ ) is larger than that in the relatively high-frequency case $(\eta=4.0)$. This is because the long waves can easily pass through the cavity to impact the ground surface. For the case of relatively short-wave incidence, the cavity will gradually become an efficient barrier. This point can be clearly seen in Fig. 4(b) that the motion amplitude on the curved cavity surface in the shaded area $\left(90^{\circ}<\theta_{1}<270^{\circ}\right)$ for high-frequency case is smaller than that for low-frequency case.

For horizontal incidence $\left(\alpha=90^{\circ}\right)$ in Fig. 5(a), motions on the ground surface over the cavity $(-1.0<x / a<1.0)$ change fast with alternatively sharp peaks and dips. This is due to the multiple wave reflections between the ground surface and top rim of the cavity. In Fig. 5(b), it is easy to distinguish between the illuminated and shaded areas on the curved cavity surface. The former ranges from about $\theta_{1}=180^{\circ}$ to $306.9^{\circ}$, while the latter ranges from about $\theta_{1}=53.1^{\circ}$ to $180^{\circ}$.

From Figs. 4(c) and 5(c), the displacement amplitudes on the flat cavity surface seem to decrease with increasing frequency, and they change gently as compared to those on the horizontal ground surface and curved cavity surface.

Next, for the sake of demonstrating the effect of the dimensionless cavity depth $d / a$ on the displacement amplitude $|u|$ on the ground surface, the ground motion responses for $d / a=2.0$ and 6.0 are calculated and shown in Fig. 6 .

As can be seen in Figs. 6(a)-(d), the vibration amplitudes of motion patterns are reduced in the relatively deep cases $(d / a=6.0)$. This may due to the fact that increasing the dimensionless cavity depth $d / a$ will lead to a reduction in interference effect of multiple reflected wave trains radiating from the ground and cavity surfaces. Results given in Fig. 6 agree with the experience from the case histories of damage to underground facilities (e.g. [5, 16]), that is to say; deeply buried structures appear to be safer and less vulnerable than shallowly buried structures.

\section{3) Spectral Variations in Ground Surface Motions}

Since completing this work, the authors have become aware of one paper [17] published much recently. For an embedded circular cavity, they presented an expression (see Eq. 33 of [17]), that is,

$$
\eta_{n}=n \frac{a}{d} \quad \text { for } \quad n \geq 1
$$

to characterize the higher harmonics of spectral variation in ground surface motion under vertical incidence. For this reason, the authors focus here on this point to display the effects of dimensionless truncation thickness $h / a$. 


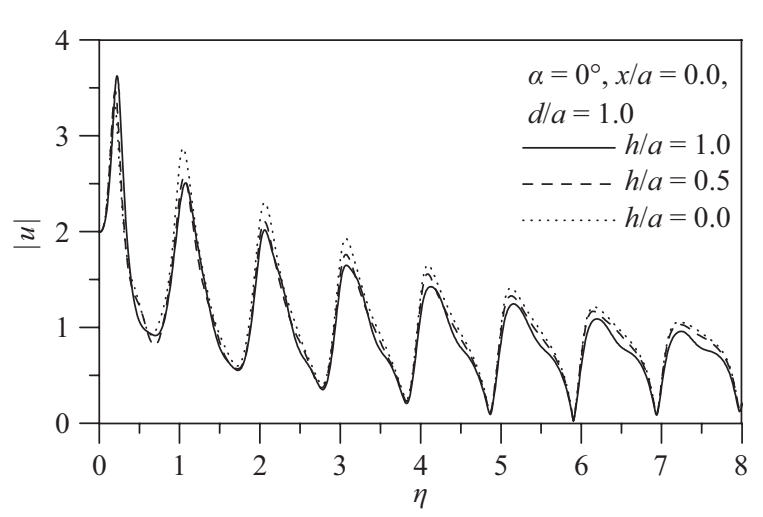

(a) $d / a=1.0$

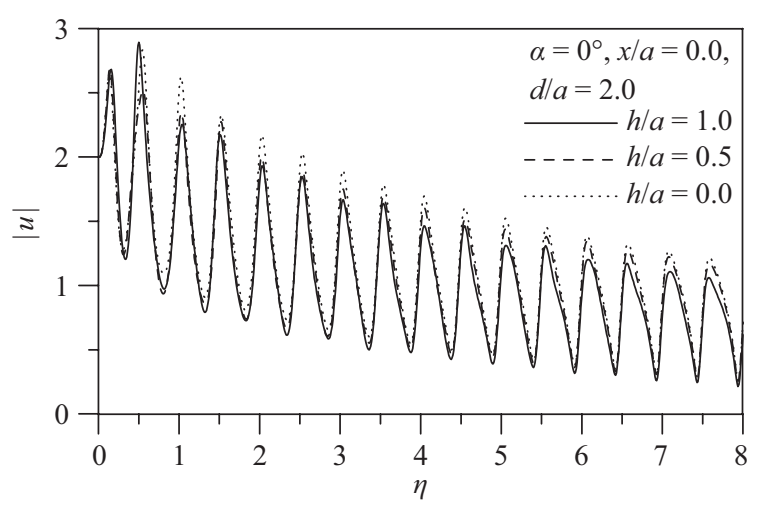

(b) $d / a=2.0$

Fig. 7. Spectral variations in ground surface motions at the position $x / a=$ 0.0 under vertical incidence $\left(\alpha=0^{\circ}\right)$.

For three cases of a circular cavity $(h / a=0.0)$, a truncated circular cavity $(h / a=0.5)$ and a semi-circular cavity $(h / a=$ 1.0), Fig. 7 gives the spectral variation in ground surface motion at the position $x / a=0.0$ under vertical incidence $\left(\alpha=0^{\circ}\right)$. As seen from Fig. 7, the amplitudes of local maximums of the spectral variation increase with decreasing dimensionless truncation thickness $h / a$ except first few peaks. The positions of these local peaks seem to be not sensitive to the change in dimensionless truncation thickness h/a. Hence, Eq. (19) still works well to predict the peak locations of ground surface motion. The main reason may be because the truncation segment is on the lower part of the cavity (i.e. on the illuminated side) so that the influence of its change on the ground surface motion at the position $x / a=0.0$ (which is on the shaded side) is small for the case of vertical incidence.

\section{Time-Domain Responses}

In this subsection, forward modeling in the frequency domain can be used to produce time-domain responses via the Fast Fourier Transform technique. The incident signal is a symmetric Ricker wavelet with the characteristic frequency $f_{c}=1.5 \mathrm{~Hz}$. The calculated frequencies are 192 in total, ranging from 0.0 to $6.0 \mathrm{~Hz}$ with $0.03125 \mathrm{~Hz}$ interval. The time window is chosen as $32 \mathrm{~s}$. The cavity radius $a$ is set to be 1.0 $\mathrm{km}$. The truncation thickness $h$ is $0.4 \mathrm{~km}$. The cavity depth $d$

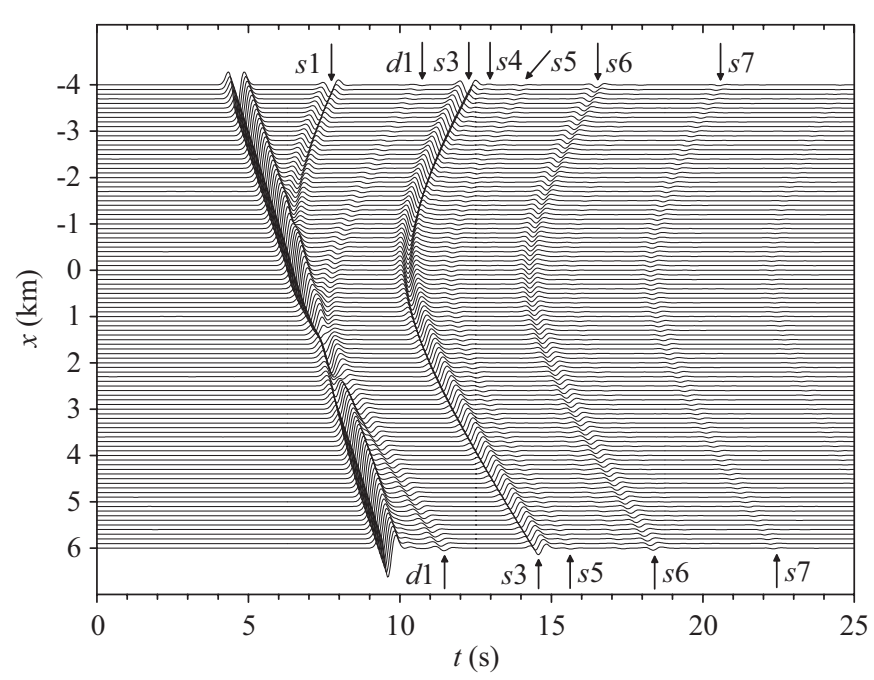

Fig. 8. Synthetic seismogram for $d / a=2.0$ at $\alpha=30^{\circ}$.

is $2.0 \mathrm{~km}$. The shear wave velocity of the half-space $c_{s}$ is $1 \mathrm{~km}$ $\mathrm{s}^{-1}$.

The synthetic seismogram for $d / a=2.0$ at oblique incidence ( $\alpha=30^{\circ}$ ) is plotted in Fig. 8. Furthermore, for the transient displacement field distributions underground, Fig. 9 illustrates a chain of snapshots at selected instants of time.

As seen in Fig. 8, some signals (labeled by arrows $d 1, s 1, s 3$, $s 4$, etc.) of scattered and diffracted waves from the cavity surface are received after the direct-wave signal. These waves bounce back and forth several times with attenuated amplitudes between the horizontal ground and cavity surfaces. Due to the first impact of the incident and specularly reflected pulses against the cavity face, the amplitudes of signals $s 1$ and $s 3$ are larger than those of signals $d 1$ and $s 4-s 7$. In the following paragraph, the marked arrows in Fig. 8 will be interpreted in detail with the aid of Fig. 9.

From Figs. 9(a) and (b), one can see that the left-hand corner of the cavity is hit first by the incoming pulse and then the scattered wave $s 1$ is emanated. A reflected wave is generated from the flat cavity surface. In Fig. 9(c), the reflected wave from the horizontal ground surface can be observed at the position about $(-3,0)$, and the incident pulse is diffracted by the curved cavity face. The scattered wave $s 2$ is originated from the right-hand corner of cavity. In Fig. 9(d), the scattered wave $s 1$ meets up the reflected wave from the horizontal ground surface. The diffracted waves $d 1$ become more obvious in about 1 o'clock direction from the cavity center. From Figs. 9(c)-(e), a principal feature of diffraction is that part of the wavefront appears to bend toward the cavity and then continuously regenerate itself. One can also perceive this propagation characteristic of diffracted waves in the right lower quadrant of Figs. 9(i)-(1).

From Fig. 9(e), one can find that, at the position about $(-3$, 0 ), the scattered wave $s 1$ is reflected by the horizontal ground surface and this newly generated wave is marked by $s 11$. From Fig. 9(f), the reflection (i.e. $d 11$ ) of diffracted waves $d 1$ 


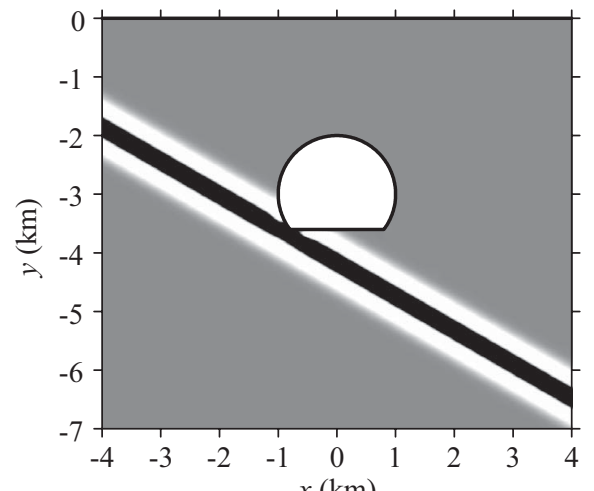

(a) $t=3.0 \mathrm{~s}$

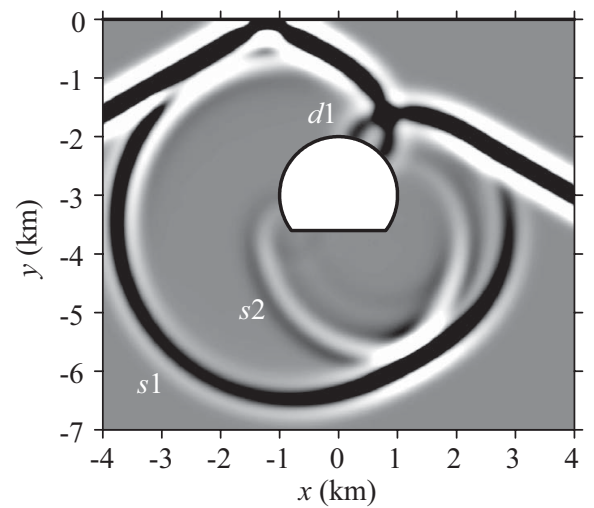

(d) $t=6.0 \mathrm{~s}$

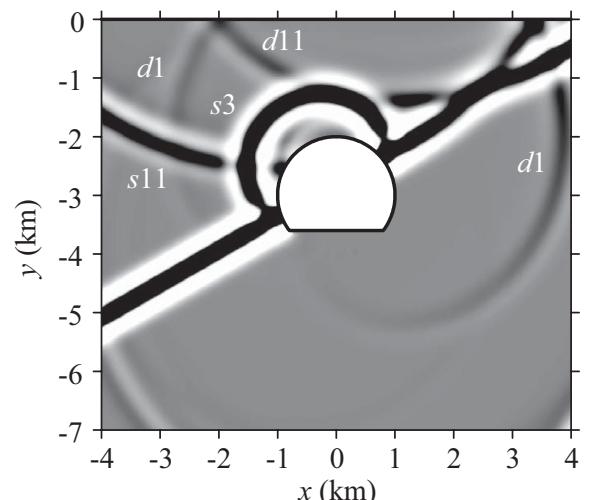

(g) $t=9.0 \mathrm{~s}$

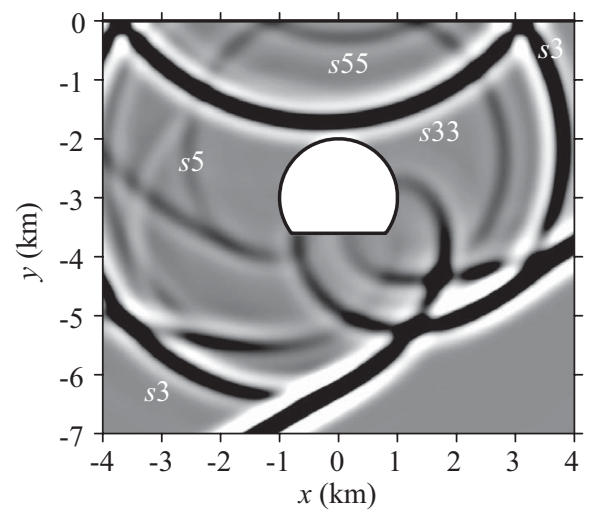

(j) $t=12.0 \mathrm{~s}$

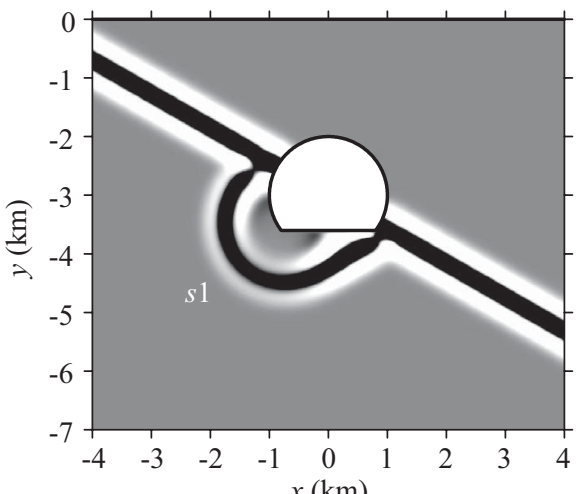

(b) $t=4.0 \mathrm{~s}$

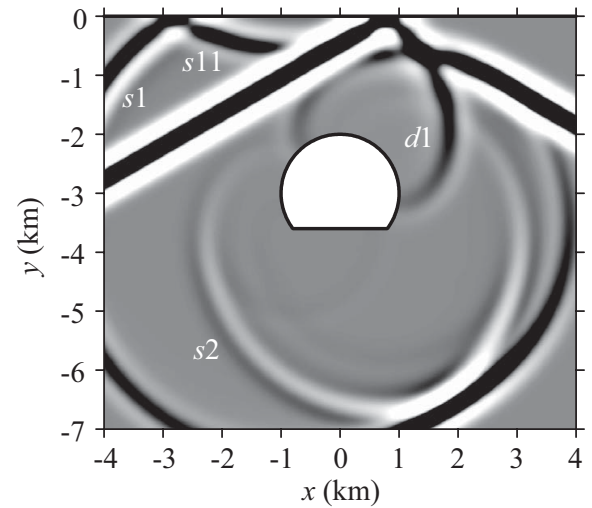

(e) $t=7.0 \mathrm{~s}$

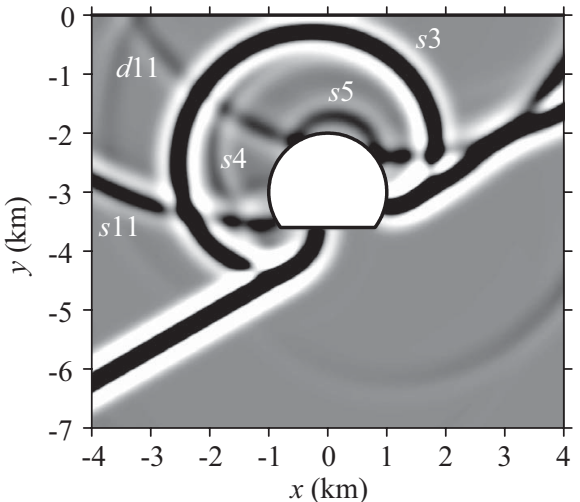

(h) $t=10.0 \mathrm{~s}$

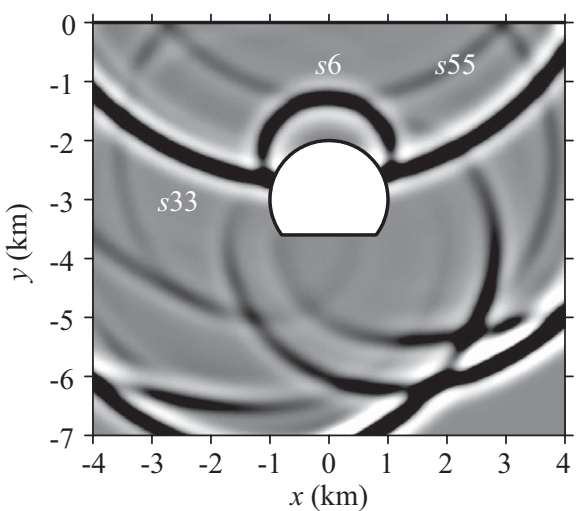

(k) $t=13.0 \mathrm{~s}$

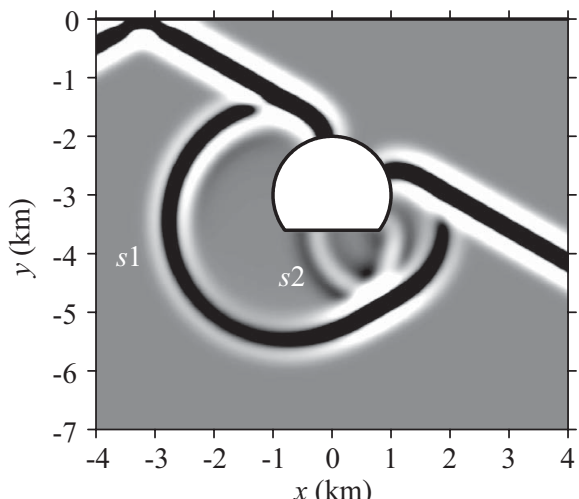

(c) $t=5.0 \mathrm{~s}$

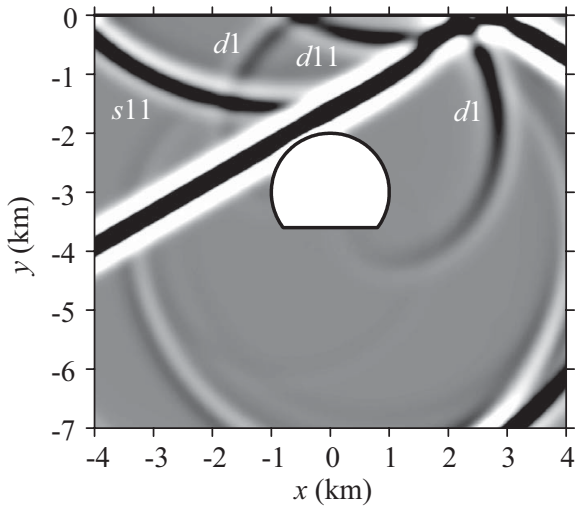

(f) $t=8.0 \mathrm{~s}$

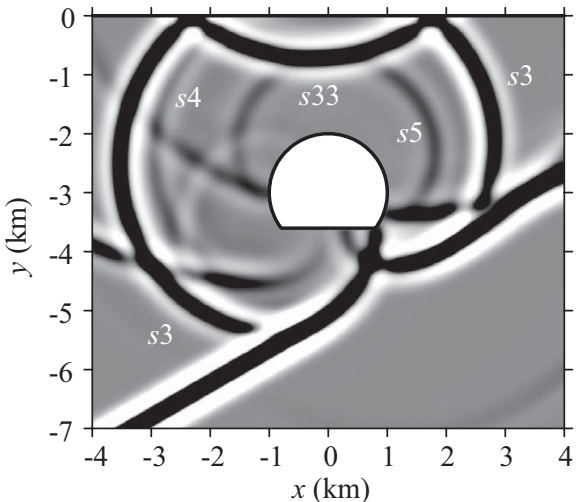

(i) $t=11.0 \mathrm{~s}$

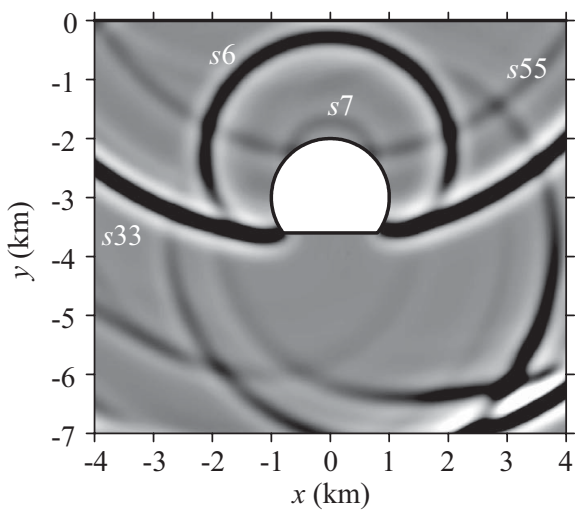

(1) $t=14.0 \mathrm{~s}$

Fig. 9. Snapshots for $d / a=2.0$ and $\alpha=30^{\circ}$ at twelve specified times. 
by the horizontal ground surface can be found at the position about $(-5,0)$. The left upper rim of the cavity is struck by the reflected wave from the ground surface, and subsequently a new scattered wave $s 3$ is radiated, see Fig. $9(\mathrm{~g})$.

In Fig. 9(h), the reflected waves $s 11$ and $d 11$ impinge the cavity face, and the corresponding scattered waves are labeled by $s 4$ and $s 5$, respectively. In Figs. 9(i) and (j), the scattered waves $s 3$ and $s 5$ are reflected by the horizontal ground surface, and the corresponding reflected waves travelling downwards are marked by $s 33$ and $s 55$, respectively. In Figs. 9(k) and (1), the scattered waves $s 6$ and $s 7$ radiated from the cavity are marked.

Overall, the interferences between the incident, reflected, diffracted and scattered wavefields are complicated. Nevertheless, the snapshots of time-domain underground motions shown in Fig. 9 encourage better understanding of the reflection, scattering and diffraction around a subsurface truncated circular cavity for an incident plane SH pulse. In addition, how they travel is visualized explicitly.

\section{CONCLUSION}

A novel series solution to the multiple scattering problem of an embedded horizontally-truncated circular cavity impinged upon by incident plane SH waves has been derived. The solution method was based on the region-matching technique, cooperating with the method of images and appropriate Graf's addition formulas. The frequency-domain responses of ground and cavity surfaces have been analysed as well as the time-domain surface and subsurface displacement distributions. Such fruitful results not only constitute a challenging testbed for the validation of numerical approximation approaches, but also provide helpful reference materials for the seismic response analysis of real underground unlined tunnels. Furthermore, the derived series solution enriches the records of known geometrical shapes for embedded cavities. The proposed region-matching technique promotes the increase of the number of geometric shapes in which the method of separation of variables cannot be used in the past. Besides, the simplified model conducted herein can be extended straightforwardly to other configurations under SH-wave incidence, such as the case of an embedded polygonal cavity; of course, its derivation will become more complicated.

\section{REFERENCES}

1. Abramowitz, M. and Stegun, I. A., Handbook of Mathematical Functions, with Formulas, Graphs, and Mathematical Tables, Dover, New York (1972).

2. Benites, R., Aki, K., and Yomogida, K., "Multiple scattering of SH waves in 2-D media with many cavities," Pure and Applied Geophysics, Vol. 138, pp. 353-390 (1992).

3. Bouchon, M., "Diffraction of elastic waves by cracks or cavities using the discrete wavenumber method," Journal of the Acoustical Society of America, Vol. 81, pp. 1671-1676 (1987).

4. Datta, S. K. and Shah, A. H., "Scattering of SH-waves by embedded cavities," Wave Motion, Vol. 4, pp. 265-283 (1982).

5. Hashash, Y. M. A., Hook, J. J., Schmidt, B., and Yao, J. I.-C., "Seismic design and analysis of underground structures," Tunnelling and Underground Space Technology, Vol. 16, pp. 247-293 (2001).

6. Koyama, Y., "Present status and technology of shield tunneling method in Japan," Tunnelling and Underground Space Technology, Vol. 18, pp. 145159 (2003).

7. Lee, S. and Kallivokas, L. F., "Local absorbing boundaries of elliptical shape for scalar wave propagation in a half-plane," Finite Elements in Analysis and Design, Vol. 40, pp. 2063-2084 (2004).

8. Lee, V. W., "On deformations near circular underground cavity subjected to incident plane SH waves," Proceeding of the Applications of Computer Methods in Engineering Conference, Los Angeles, pp. 951-962 (1977).

9. Lee, V. W., Chen, S., and Hsu, I. R., "Anti-plane diffraction from canyon above a subsurface unlined tunnel," ASCE Journal of Engineering Mechanics, Vol. 125, pp. 668-675 (1999).

10. Lee, V. W. and Manoogian, M. E., "Surface motion above an arbitrary shape underground cavity for incident SH waves," European Earthquake Engineering, Vol. 8, pp. 3-11 (1995).

11. Liao, W. I., Yeh, C. S., and Teng, T. J., "Scattering of elastic waves by a buried tunnel under obliquely incident waves using T matrix," Journal of Mechanics, Vol. 24, pp. 405-418 (2008).

12. Liu, P. and Liang, W. H., "Design considerations for construction of the Qinling Tunnel using TBM," Tunnelling and Underground Space Technology, Vol. 15, pp. 139-146 (2000).

13. Luco, J. E. and de Barros, F. C. P., "Dynamic displacements and stresses in the vicinity of a cylindrical cavity embedded in a half-space," Earthquake Engineering \& Structural Dynamics, Vol. 23, pp. 321-340 (1994).

14. Pao, Y. H. and Mow, C. C., Diffraction of Elastic Waves and Dynamics Stress Concentrations, Crane, Russak and Company Inc., New York (1973).

15. Shah, A. H., Wong, K. C., and Datta, S. K., "Diffraction of plane SH waves in a half-space," Earthquake Engineering \& Structural Dynamics, Vol. 10, pp. 519-528 (1982).

16. Sharma, S. and Judd, W. R., "Underground opening damage from earthquakes," Engineering Geology, Vol. 30, pp. 263-276 (1991).

17. Smerzini, C., Avilés, J., Paolucci, R., and Sánchez-Sesma, F. J., "Effect of underground cavities on surface earthquake ground motion under $\mathrm{SH}$ wave propagation," Earthquake Engineering \& Structural Dynamics, Vol. 38, pp. 1441-1460 (2009).

18. Tsaur, D. H. and Chang, K. H., "Scattering of SH waves by truncated semicircular canyon," ASCE Journal of Engineering Mechanics, Vol. 135 No. 8, pp. 862-870 (2009).

19. Tsaur, D. H. and Chang, K. H., "SH-waves scattering from a partially filled semi-circular alluvial valley," Geophysical Journal International, Vol. 173, No. 1, pp. 157-167 (2008).

20. Uchida, K., Wasa, Y., and Kanal, M., "Design of the shield tunnel for the Trans-Tokyo Bay Highway," Tunnelling and Underground Space Technology, Vol. 7, pp. 251-261 (1992).

21. Varadan, V. V., Ma, Y., Varadan, V. K., and Lakhtakia, A., "Scattering of waves by spheres and cylinders," in: Varadan, V. V., Lakhtakia, A., and Varadan, V. K. (Eds), Field Representations and Introduction to Scattering, North-Holland, Amsterdam, pp. 211-324 (1991).

22. Watson, G. N., A Treatise on the Theory of Bessel Functions, 2nd Ed., Cambridge University Press, Cambridge, England (1958).

23. Yu, C.-W. and Dravinski, M., "Scattering of a plane harmonic SH wave by a completely embedded corrugated scatterer," International Journal for Numerical Methods in Engineering, Vol. 78, pp. 196-214 (2009). 